\title{
EFECTO DEL CITRATO DE SILDENAFIL EN LA PROGRESIÓN DE LA ENFERMEDAD HEPÁTICA CRÓNICA EN UN MODELO DE EXPERIMENTACIÓN MURINO
}

\section{EFFECT OF SILDENAFIL CITRATE IN CHRONIC LIVER DISEASE PROGRESSION IN A MURINE EXPERIMENTAL MODEL}

\begin{abstract}
Jorge Cruz A. ${ }^{1}$, Juan Pérez Z. ${ }^{2}$, Martha Urrea Q. ${ }^{3}$, Henry Mesa E. ${ }^{4}$
${ }^{1}$ MV, Ph.D. Docente Departamento de Salud Animal, Facultad de Ciencias Agropecuarias. Universidad de Caldas calle 65 $\mathrm{N}^{\circ}$ 26-10. Manizales, Colombia; ${ }^{2}$ MVZ, Candidato MSc, Facultad de Ciencias Agropecuarias. Universidad de Caldas; ${ }^{3}$ MV, Candidato Esp. Docente Departamento de Salud Animal, Facultad de Ciencias Agropecuarias. Universidad de Caldas; ${ }^{4}$ MVZ, PhD. Docente Departamento de Producción. Facultad de Ciencias Agropecuarias. Universidad de Caldas. Dirección Correspondencia: E-mail: mario.cruz@ucaldas.edu.co; juanperezzapata@gmail.com; henry.mesa@ucaldas.edu.co; marthaisabeluq@hotmail.com
\end{abstract}

Rev. U.D.C.A Act. \& Div. Cient. 16(1): 53 - 60, 2013

\section{RESUMEN}

El tratamiento médico de la cirrosis es de carácter paliativo, siendo el trasplante de hígado la única opción de curación a largo plazo, aunque poco disponible. No se ha establecido un régimen terapéutico eficaz que permita evitar la progresión a cirrosis. Se evaluó el efecto del citrato de sildenafil (CS) en la fibrogénesis de ratas Wistar con daño hepático, inducido con tetracloruro de carbono (CCL4). El proyecto fue avalado por el comité de ética para la experimentación con animales de la Universidad de Caldas. Se distribuyeron siete tratamientos: Control (sin medicamentos); control CCL4; CS $10 \mathrm{mg} / \mathrm{kg}$; CS $25 \mathrm{mg} / \mathrm{kg}$; CS 50mg/kg; CCL4 + CS 10mg/kg; CCL4 + CS $25 \mathrm{mg} / \mathrm{kg}$; CCL4 + CS 50mg/kg. El CCL4, se administró dos veces por semana vía subcutánea (SC), mientras que el CS, tres veces por semana, vía intraperitoneal (IP). Se cuantificó la enzima aspartato aminotransferasa (AST) y se realizó histopatología cada dos semanas. El análisis de resultados, se efectuó mediante un análisis de varianza unifactorial. El nivel de significancia estadística fue tomado como $\mathrm{P}<0.05$. Se encontró que los tratamientos CCL4 + CS tenían incrementos importantes de la AST, siendo la dosis $10 \mathrm{mg} / \mathrm{kg}$ la que presentó un aumento estadísticamente significativo $(P<0.05)$. No se observaron diferencias significativas en la evaluación histopatológica de las variables infiltración celular, vacuolización y fibrosis, al comparar los tratamientos CCL4 + CS y su respectivo grupo control. El tratamiento con CS no alteró la progresión de la fibrogénesis hepática y aumentó de manera significativa los niveles de AST, sugiriendo un posible efecto tóxico.

Palabras clave: CCL4, fibrogénesis hepática, histopatología, AST.

\section{SUMMARY}

Medical treatment of cirrhosis is palliative, being the hepatic transplant the only long-term cure but although not an accessible alternative. No effective therapeutic regimen that could prevent the progression of cirrhosis has been established. The effect of the sildenafil citrate (SC) was evaluated in Wistar rats with hepatic damage induced with carbon tetrachloride (CCL4). The project had the support of the ethic committee of the University of Caldas to experiment with animals. The animals were distributed in seven treatments. Normal control (No medicine); control CCL4; CS $10 \mathrm{mg} / \mathrm{kg} ;$ SC $25 \mathrm{mg} / \mathrm{kg}$; SC 50mg/kg; CCL4 + SC $10 \mathrm{mg} / \mathrm{kg} ;$ CCL4 + SC $25 \mathrm{mg} / \mathrm{kg}$; CCL4 + SC $50 \mathrm{mg} / \mathrm{kg}$. The CCL4 was given twice a week through subcutaneous route, meanwhile the SC was administered within the peritoneum (IP) route three times a week. Samples for the plasmatic measurement were taken of the aspartato aminotransferase (AST), as well as the histopathology tests every two weeks. The analysis of the results was done through an analysis of unifactorial variance. The level of statistical significance was taken as $\mathrm{P}<0.05$. It was found that treatments of CCL4 + 
SC showed important increases of the enzyme AST, being the dose $10 \mathrm{mg} / \mathrm{kg}$ the one with a statistically significant increase $(\mathrm{P}<0.05)$. Significant differences were not observed in the histopathology evaluation of the variables of cellular infiltration, vacuolization and fibrosis when comparing the treatments CCL4 + SC with its respective control group. The treatment with SC did not affect the progression of the hepatic fibrogenesis and noticeably increased the levels of AST, suggesting a possible toxic effect.

Key words: CCL4, hepatic fibrogénesis, histopatology, AST.

\section{INTRODUCCIÓN}

Un porcentaje importante (2-3\%) de la población mundial sufre algún tipo de enfermedad hepática crónica, caracterizada por fibrosis progresiva y cirrosis, como resultado final (Ji et al. 2005).

La cirrosis hepática tiene un gran impacto clínico, debido a que: 1) 170 millones de pacientes alrededor del mundo se encuentran afectados por alguna forma de enfermedad hepática crónica (EHC) y, dentro de este grupo, el 25 $30 \%$ es de esperar que desarrollen fibrosis y eventualmente cirrosis; 2) la cirrosis hepática es, entre las enfermedades del tracto gastrointestinal, la causa no neoplásica más común de muerte en Europa y en USA y representa la séptima causa de muerte en los países occidentales; 3 ) la mortalidad es aún mayor, debido a que la cirrosis predispone al desarrollo de carcinoma hepatocelular ( $\mathrm{CHC}$ ), siendo el quinto cáncer más frecuente y la tercera causa de muerte cancerígena alrededor del mundo y, 4) análisis epidemiológicos predicen un pico para las EHCs (incluyendo $\mathrm{CHC}$ ) en la próxima década, con un incremento en los pacientes que llegarán al estado final de la enfermedad (Forbes \& Parola, 2011).

Cuando la cirrosis está bien establecida, la sobrevida del paciente no supera el año. Los tratamientos médicos disponibles para la enfermedad hepática crónica son de carácter paliativo y casi siempre son inefectivos, con el trasplante de hígado, como única opción de brindar una expectativa de vida a largo plazo, aunque esta alternativa es muy costosa y limitada a unos pocos pacientes (Albanis et al. 2003).

La activación de las células hepáticas estrelladas constituye la piedra angular de la patogenia en la fibrosis hepática. Estas células perisinusoidales son estructuras quiescentes que almacenan vitamina $A$ en condiciones normales, pero se diferencian a células contráctiles, proliferativas y fibrogénicas, productoras de matriz extracelular en exceso, en el paciente cirrótico. Los mediadores claves en la activación de células hepáticas estrelladas incluyen una serie de citoquinas, intermediarios reactivos de oxígeno y otras señales paracrinas y autocrinas (Bonis et al. 2001).
Durante la injuria hepática las concentraciones de endotelina aumentan promoviendo la proliferación de las células estrelladas. Paralelo, se presenta una reducción del óxido nítrico (ON), derivado de las células endoteliales; el ON antagoniza el efecto de la endotelina sobre las células hepáticas estrelladas. El resultado de este imbalance es una proliferación descontrolada de este tipo de células con gran liberación de matriz extracelular y un invariable incremento de la resistencia sinusoidal, que contribuye a la hipertensión portal (Iredale, 2003).

Existen opiniones controversiales respecto a la utilidad que tendría el ON en revertir la fibrogenesis hepática. Experimentos in vivo muestran que el ON tiene efectos hepatoprotectores, aunque producido en grandes cantidades o crónicamente puede actuar como un mediador citotóxico e inducir daño hepático (Mayoral et al. 1999). El ON protege al hígado inhibiendo la activación de la caspasa, a través de modificaciones de residuos de cistina en los sitios de acción de esta enzima, proceso conocido como S-nitrolización, que es particularmente eficiente en hepatocitos y células endoteliales. También puede activar la guanil ciclasa (sGC), que lidera la síntesis de guanilmonofosfato cíclico (cGMP) y tiene una actividad anti-apoptótica en los hepatocitos y es la responsable de desencadenar vasodilatación (Rockey \& Shah, 2004).

El efecto vasodilatador del ON está limitado por la enzima fosfodiesteraza 5 (PDE-5) (Bender \& Beavo, 2006). LoureiroSilva et al. (2006) afirman que la PDE-5 modula el tono intrahepático vascular en hígados normales y que su sobre expresión está involucrada en la reducción de la respuesta vasodilatadora mediada por el ON en hígados cirróticos de ratas; estos investigadores postulan que los inhibidores de la PDE-5 pueden ser útiles para reducir la resistencia intrahepática vascular incrementada en la cirrosis, debido a que potencian los efectos vasodilatadores endógenos y exógenos del ON, aumentando el flujo vascular.

El Citrato de Sildenafil (CS) un agente vasodilatador que inhibe la enzima PDE-5, lo que incrementa los niveles de ON; ha sido ampliamente utilizado para tratar la disfunción eréctil y se emplea, experimentalmente, como estrategia en el mejoramiento de la función endotelial (Tomasoni et al. 2010), en pacientes con falla cardiaca e hipertensión pulmonar reactiva (Dumitrescu et al. 2012), en la fibrosis pulmonar inducida con bleomicina en modelos murinos (Yildirim et al. 2010) y en el manejo de enfermedades autoinmunes (Shenoy \& Agarwal, 2010), problemas cardiovasculares de arterias coronarias (Reyna-Villasmil et al. 2008; Halcox et al. 2002), entre otros.

Se ha encontrado que en caninos con hipertensión pulmonar (HTP), el CS reduce la presión arterial pulmonar (PAP), 
incrementa la actividad física y mejora la calidad de vida y la supervivencia (Brown et al. 2010; Bach et al. 2006; Kellum \& Stepien, 2007), convirtiéndose en una opción razonable de tratamiento para perros que tienen HTP; sin embargo, no se conocen reportes de su uso en la enfermedad hepática en pequeños animales.

El protocolo para inducir daño hepático con tetracloruro de carbono (CCL4) ha sido ampliamente utilizado y garantiza la producción de cirrosis hepática (Proctor \& Chatamra, 1984). Este protocolo constituye uno de los métodos más confiables para evaluar el efecto terapéutico antifibrótico.

El objetivo de este trabajo fue evaluar el efecto del CS sobre los niveles de AST y la fibrogénesis en un modelo de experimentación murino de daño hepático inducido con CCL4.

\section{MATERIALES Y MÉTODOS}

Este experimento, se llevó a cabo en las instalaciones del Bioterio de la Universidad de Caldas, ubicada en la ciudad de Manizales, a 2.150 m.s.n.m., con temperatura promedio de $20^{\circ} \mathrm{C}$ y humedad relativa de $78 \%$. Los análisis histopatológicos y sanguíneos fueron realizados en los laboratorios de Patología Veterinaria y Patología Clínica de la misma institución. El proyecto fue aprobado por el comité de ética de la Universidad de Caldas.

Se utilizaron 94 ratas Wistar, con un peso inicial aproximado de $200 \mathrm{~g}$ y ocho meses de edad, procedentes del bioterio de la Universidad de Caldas. Se distribuyeron en 28 cajas plásticas transparentes $(37,5 \mathrm{~cm} \times 57,5 \mathrm{~cm} \times 37,0 \mathrm{~cm})$, bajo una temperatura constante de $26^{\circ} \mathrm{C}$ y humedad relativa de $75 \%$. El piso de cada caja, se cubrió con cascarilla de arroz como cama y se hizo recambio tres veces por semana. Los animales tuvieron acceso al agua y alimento (Agrinal ${ }^{\circledR}$ Rodentina) ad libitum, durante todo el experimento.

Los animales, se distribuyeron en siete tratamientos (Tabla 1), organizados en un Diseño Irrestrictamente al Azar, conformados por cuatro repeticiones y cuatro animales por unidad experimental.

La inducción del daño hepático, se realizó con la aplicación de Tetracloruro de Carbono (CCL4), según el protocolo de

Tabla 1. Tratamientos experimentales.

\begin{tabular}{|l|c|c|c|}
\hline \multicolumn{1}{|c|}{ Tratamiento } & CCL4 & Citrato de Sildenafil (CS) & Dosis \\
\hline T0 Control & - & - & - \\
\hline T1 CCL4 & + & - & - \\
\hline T2 Sanos + C.S. & - & + & $10 \mathrm{mg} / \mathrm{kg} \mathrm{v.p.}$ \\
\hline T3 Sanos + C.S. & - & + & $25 \mathrm{mg} / \mathrm{kg} \mathrm{v.p}$ \\
\hline T4 Sanos + C.S. & - & + & $50 \mathrm{mg} / \mathrm{kg} \mathrm{v.p}$ \\
\hline T5 CCL4 + C.S. & + & + & $10 \mathrm{mg} / \mathrm{kg} \mathrm{v.p}$ \\
\hline T6 CCL4 + C.S. & + & + & $25 \mathrm{mg} / \mathrm{kg} \mathrm{v.p}$ \\
\hline T7 CCL4 + C.S. & + & + & $50 \mathrm{mg} / \mathrm{kg} \mathrm{v.p}$ \\
\hline
\end{tabular}

- No aplica

+ Si aplica

Proctor \& Chatamra (1984); se aplicó el CCl4 mezclado con aceite de oliva, en una proporción de 1:1; esta mezcla, se inyectó tres veces por semana a la dosis de $1 \mathrm{ml} / \mathrm{kg}$ de peso corporal por vía SC, durante ocho semanas, tiempo, en el cual, se evaluó la progresión de la enfermedad hepática.

La solución de CS, se preparó según el protocolo de Behn \& Potter (2001); se utilizó una técnica aséptica, se pulverizaron tabletas de CS 50mg (Sildenafil Lab. Mk ${ }^{\circledR}$ ), con mortero y pistilo hasta obtener un fino polvo. El polvo fue mezclado con solución salina $0,9 \%$, para conseguir una solución homogénea, que se filtró con papel filtro de $40 \mu \mathrm{m}$ y se almacenó en recipientes plásticos estériles. Una vez obtenida la solución, se procedió a inyectar a los animales vía IP, tres veces por semana, a una dosis de 10, 25 y 50mg/ $\mathrm{kg}$, de acuerdo al tratamiento correspondiente, durante un periodo de seis semanas y a partir de la segunda semana de recibir el tóxico. Los animales fueron pesados previamente a la aplicación del medicamento para asegurar una dosis apropiada del mismo.

En las semanas cuatro, seis y ocho, se sacrificó un animal por unidad experimental. La eutanasia, se realizó con una sobredosis de Isofuorano (Isorane Lab. Abbot ${ }^{\circledR}$ ), según lo 
establecido por la American Veterinary Association (2007). Una vez logrado el paro cardiorrespiratorio, se tomó la muestra de sangre por punción intracardiaca y se depositó en un tubo sin anticoagulante (Kenxin ${ }^{\circledR}$ x $6 \mathrm{ml}$ ). Posteriormente, se hizo necropsia y se tomó una muestra de hígado, para el estudio histopatológico. Se registraron los hallazgos macroscópicos. Para la preservación de las muestras, se utilizó formol buferado al $10 \%$.

Las muestras de sangre, se centrifugaron a $2000 \mathrm{rpm}$, durante 15 minutos, para la obtención de suero. Se realizó la medición de la enzima aspartato aminotransferasa (AST) en un espectrofotómetro Biosystem ${ }^{\circledR}$.

Las muestras de hígado fueron teñidas con hematoxilinaeosina. Para realizar la lectura de las placas de histopatología, se tuvo en cuenta el grado de actividad o severidad de la inflamación, la vacuolización y la fibrosis, en una escala de valores de cero a tres, en donde cero representa ninguna actividad y tres, actividad necroinflamatoria masiva (Tabla 2), según lo reportado por Cruz-Amaya et al. (2011) y se adaptó el sistema marcador METAVIR (Theise, 2007).

Tabla 2. Calificación de los estadios del daño hepático.

\begin{tabular}{|l|c|c|c|c|}
\hline \multicolumn{1}{|c|}{ Ítem } & $\mathbf{0}$ & $\mathbf{1}$ & $\mathbf{2}$ & $\mathbf{3}$ \\
\hline Actividad inflamatoria & Ninguna & Leve & Moderada & Severa \\
\hline Vacuolización & Ninguna & Leve & Moderada & Severa \\
\hline Fibrosis & Ninguna & Leve & Moderada & Severa \\
\hline
\end{tabular}

Las diferencias estadísticas entre grupos de tratamiento fueron determinadas, utilizando un análisis de varianza unifactorial. El nivel de significancia estadística fue tomado como $\mathrm{P}<0.05$.

\section{RESULTADOS Y DISCUSIÓN}

En este trabajo ningún tratamiento alteró la progresión de la fibrogénesis hepática inducida por el CCL4 y no se observaron diferencias significativas en la evaluación histopatológica de las variables infiltración celular, vacuolización y fibrosis al comparar los tratamientos CCL4 + CS en las dosis de 10, 25 y $50 \mathrm{mg} / \mathrm{kg}$ con su respectivo grupo control (Figura 1 ).

La evaluación histopatológica realizada, según lo reportado por Cruz-Amaya et al. (2011), mostró marcada a severa vacuolización acompañada de degeneración grasa o hidrópica centrolobulillar, así como fibrosis centrolobulillar y puentes fibróticos de comunicación porto-central en todos los tratamientos, en los cuales, el CCL4 estaba presente. Estos hallazgos fueron más notorios en el tratamiento CCL4 $+10 \mathrm{mg} / \mathrm{kg}$ de CS (Figura 1).

Por otra parte, al evaluar el efecto de tratamiento sobre la enzima plasmática AST, se encontró que los tratamientos de CCL4 y CS en las dosis de 10, 25 y 50mg/kg tenían incrementos importantes de la enzima, siendo la dosis 10 $\mathrm{mg} / \mathrm{kg}$ de CS la que presentó un aumento estadísticamente significativo (Figura 2), indicando, posiblemente, que el suministro del fármaco junto con CCL4 produjo un sinergismo de potenciación tóxica.
En este experimento, no se observaron signos clínicos característicos de cirrosis, como ascitis o ictericia; en general, la población evaluada no presentó signos de enfermedad. De igual forma, no se evidenciaron diferencias estadísticamente significativas en las variables peso y consumo de alimento.

Existe una experiencia clínica considerable con el uso del CS, como vasodilatador en situaciones diversas, que incluyen la disfunción eréctil y la hipertensión pulmonar (Corbin et al. 2002; Oliver et al. 2006; Spring et al. 2008), lo que ha generado gran interés en establecer si este fármaco puede contrarrestar la hipertensión portal y la fibrogénesis en el paciente cirrótico.

Una gran controversia gira en torno al hecho de manipular terapéuticamente los niveles de $\mathrm{ON}$ en el paciente cirrótico, debido a que estos niveles estarían disminuidos a nivel hepático en dichos pacientes, pero no a nivel sistémico y un aumento de esta molécula en toda la economía esta asociada, al parecer, a mayor deterioro hepático (Atucha et al. 2005).

En este trabajo, la administración IP de CS no alteró la progresión de la fibrogénesis hepática. Por el contrario, algunos investigadores consideran que la administración oral de fármacos que incrementan los niveles de ON puede modificar no solamente el aumento de la resistencia hepática sino también ejercer una acción antifibrogénica por inhibición de la proliferación, la motilidad y la contractilidad de las células hepáticas estrelladas, reduciendo la acumulación de matriz extracelular fibrilar y retrasando la progresión de la 


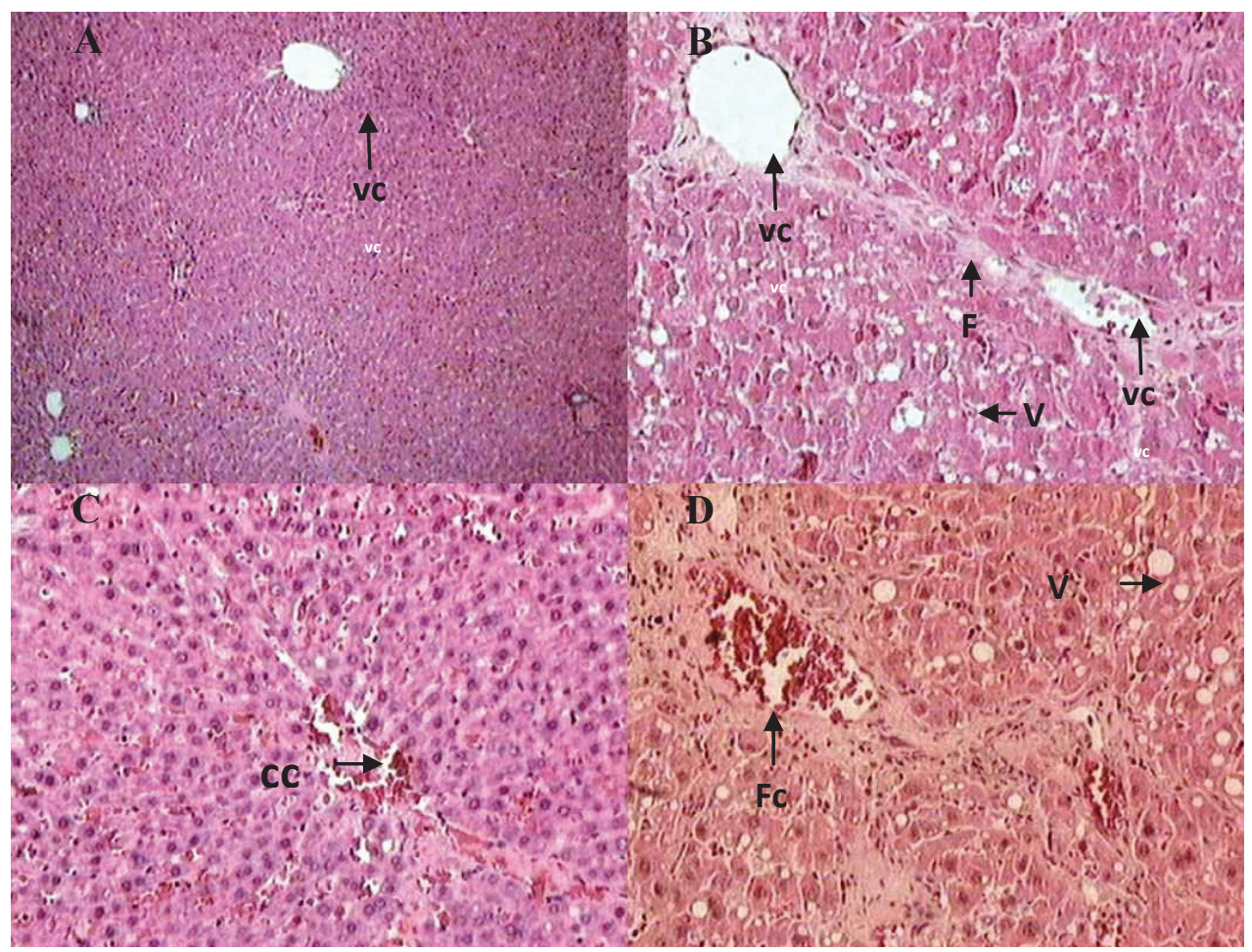

Figura 1. Hallazgos histopatológicos - semana 8 de tratamiento. (A) Control T0, SE puede observar el hígado normal (magnificación 100x). (B) Control CCl4, se observa fibrosis (F) uniendo venas centrales (vc) con moderada a severa vacuolización (V) centrolobulillar (magnificación 400x). (C) Tratamiento T2, leves cambios microcirculatorios asociados a congestión centrolobulillar (cc) (magnificación 400x). (D) Tratamiento T5, moderados cambios microcirculatorios y grasos o hidrópicos, hepatopatía crónica con severa degeneración grasa y fibrosis centrolobulillar (Fc) (magnificación 400x).

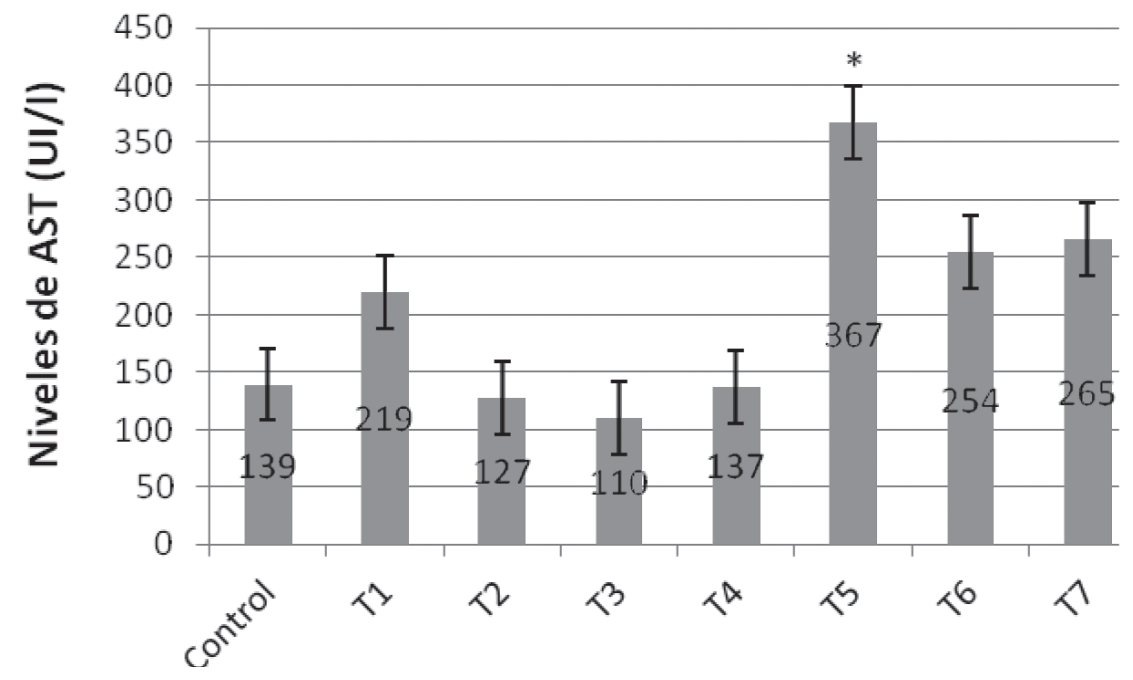

Figura 2. Niveles plasmáticos de AST en cada tratamiento (valores normales 96 -153 Ul/l). *Diferencia estadísticamente significativa $(\mathrm{P}=0.000002)$. 
cirrosis (Bosch et al. 2003; Failli et al. 2000); no obstante, en este trabajo, los cambios histopatológicos en los animales tratados con CS ip no evidenciaron disminución en la fibrogénesis, al compararlos con su respectivo grupo control (Figura 1).

Loureiro-Silva et al. (2006) describen que en el hígado cirrótico existe una sobreexpresión de la PDE-5, enzima que limita el efecto vasodilatador del $\mathrm{ON}$ al degradar selectivamente la guanosina monofosfato cíclica (GMPc), la cual, en condiciones normales es activada por el ON, lo que resulta en una secuencia de eventos que culminan con la relajación y la vasodilatación endotelial.

Por estas razones, se cree que la terapia con el CS al incrementar los niveles de ON, podría representar una estrategia en el tratamiento de la cirrosis, lo que favorece a la reducción de la resistencia intrahepática vascular incrementada y el gradiente de presión vascular hepático (GPVH), al potenciar los efectos vasodilatadores endógenos del ON, aumentando el flujo vascular (Tandon et al. 2010; Ghali-Ghoul et al. 2007; Loureiro-Silva et al. 2006); no obstante, los efectos fisiológicos de los fármacos inhibidores de la FDE-5, como el CS, no se pueden considerar de forma simplista, debido a que al parecer los niveles de esta molécula difieren notablemente entre el lecho vascular hepático y el sistémico.

Rodríguez-Vilarrupla et al. (2007) y Atucha et al. (2005) reportan que, a nivel esplácnico y sistémico, el paciente cirrótico experimenta un exceso de $\mathrm{ON}$, que incrementa el flujo sanguíneo hacia la vena porta y agrava el síndrome hipertensivo portal. De otra parte, Colle et al. (2004) demostraron que el CS administrado intramesentérico e intravenoso además de incrementar el flujo de sangre mesentérico y la presión venosa portal, disminuye la presión arterial en ratas control y en ratas con cirrosis, lo que tendría posibles implicaciones clínicas, como una ruptura de varices esofágicas, debido a un súbito aumento de la presión venosa portal.

Además, la hipotensión causada por el CS puede ser deletérea en pacientes con cirrosis, los cuales, ya presentan una presión sanguínea disminuida y, por último, un aclaramiento retrasado del CS en plasma puede incrementar los efectos secundarios, ya mencionados (Colle et al. 2004).

Tandon et al. (2010) reportan que en pacientes con cirrosis hepática compensada, el suministro de CS no tuvo un efecto benéfico sobre la reducción de la presión portal y que la acción vasodilatadora del CS al no ser hepático específica, dio como resultado una reducción significativa en la presión arterial media (PAM).

A la luz de las conclusiones de Tandon et al. (2010) y
Rodríguez-Vilarrupla et al. (2007), se podría pensar que el suministro de CS a un paciente cirrótico conseguiría generar un aumento en la circulación portal lo suficientemente severo, para agravar el síndrome hipertensivo.

El aumento en la AST se considera un indicador de injuria hepática activa. En este trabajo, los niveles de la enzima AST se incrementaron en los tratamientos que incluían CCL4 y CS. La asociación en estos hallazgos sugiere que el CS favorece al deterioro microscópico en los hígados de las ratas con enfermedad hepática inducida con CCL4; estos hallazgos coinciden con lo reportado por Li et al. (2005), investigadores que encontraron un sinergismo de potenciación cuando se suministra CS y etanol concomitantemente.

Ji et al. (2005) cuantificaron los niveles séricos de bilirrubina total y AST para establecer si se producía mejoría en la función y en la regeneración hepática de ratas alimentadas con y sin alcohol, sometidas a hepatectomía parcial, medicadas con CS, a dosis de 5,25 y $50 \mathrm{mg} / \mathrm{kg}$ vía oral. Los investigadores no observaron cambios significativos en la funcionalidad hepática en ambos grupos de ratas; por el contrario, encontraron que la dosis de $5 \mathrm{mg} / \mathrm{kg}$ inhibía la regeneración hepática. De otra parte, Yardimci et al. (2012) consideran que las dosis utilizadas por Ji et al. (2005), fueron muy altas, ya que ellos encontraron regeneración hepática con el mismo modelo experimental, pero a dosis mucho menores $(10 \mu \mathrm{g} / \mathrm{kg}$ y $100 \mu \mathrm{g} / \mathrm{kg})$.

Es también muy significativo el hallazgo de Yardimci et al. (2012), respecto al hecho de que el CS no solamente produjo regeneración hepática sino que también esta regeneración fue mucho más temprana, con respecto al grupo control.

Edwards et al. (2008) sugieren que el ideal para una intervención segura y efectiva en el tratamiento de la hipertensión portal debe ser un fármaco donador de ON hepático específico que pueda reducir la presión venosa portal sin afectar la hemodinamia sistémica. Sumado a lo anterior, Tandon et al. (2010) afirman que la demostración de un incremento en la expresión de la FDE-5 en hígados cirróticos de ratas, apunta al desarrollo de inhibidores de la enzima específicos para el órgano, lo que conseguiría una reducción del GPVH sin inducir efectos sistémicos.

El suministro de CS a ratas con daño hepático inducido con CCL4 no tuvo efecto en la reversión de la fibrogénesis hepática. Además, el aumento significativo de los niveles plasmáticos de AST sugiere un efecto tóxico del CS.

Posiblemente, los experimentos futuros que evalúen la terapia del CS u otros inhibidores de la enzima FDE - 5 en pacientes cirróticos deban considerar una vía de administración que no exponga la economía a niveles incrementados de esta 
molécula sino exclusivamente la circulación hepática, así como también la utilización de otras dosis de este medicamento.

Se requieren estudios adicionales sobre vasodilatadores intrahepáticos específicos, que no tengan efectos sobre la circulación sistémica.

Agradecimientos: A la Vicerrectoría de Investigaciones y Posgrados de la Universidad de Caldas, por patrocinar esta investigación. Conflictos de intereses: El manuscrito fue preparado y revisado con la participación de todos los autores, quienes declaramos que no existe ningún conflicto de intereses que ponga en riesgo la validez de los resultados presentados.

\section{BIBLIOGRAFÍA}

1. ALBANIS, E.; SAFADI, R.; FRIEDMAN, S. 2003. Treatment of Hepatic Fibrosis: Almost There. Curr. Gastroen. Report (Estados Unidos). 5:48-56.

2. AMERICAN VETERINARY MEDICAL ASOCIATION (AVMA). 2007. Guidelines on euthanasia. Disponible en: http://www.avma.org/issues/animal_welfare/ euthanasia.pdf (con acceso 20/03/2010).

3. ATUCHA, N.; NADAL, F.; IYÚ, D.; ALCARAZ, A.; RODRÍGUEZ-BARBERO, A.; ORTIZ, C.; LÓPEZNOVOA, M.; GARCÍA-ESTAÑO, J. 2005. Role of vascular nitric oxide in experimental liver cirrhosis. Curr. Vasc. Pharmacol. (Emiratos Árabes Unidos). $3(1): 81-5$.

4. BACH, J.F.; ROZANSKI, EA.; MACGREGOR, J.; BETKOWSKI, J.M.Y.; RUSH, J.E. 2006. Retrospective evaluation of sildenafil citrate as a therapy for pulmonary hypertension in dogs. J. Vet. Intern. Med. (Estados Unidos). 20(5):1132-1135.

5. BEHN, D.; POTTER, M. 2001. Sildenafil-mediated reduction in retinal function in heterozygous mice lacking the gamma-subunit of phosphodiesterase. Invest. Ophthalmol. Vis. Sci. (Estados Unidos). 42(2):523-527.

6. BENDER, A.T.; BEAVO, J.A. 2006. Cyclic nucleotide phosphodiesterases: molecular regulation to clinical use. Pharmacol. Rev. (Estados Unidos). 58(3):488520.

7. BOSCH, J.; ABRALDES, J.; GROSZMANN, R. 2003. Current management of portal hypertension. J. Hepatol. (Paises Bajos). 38 S54-S68.
8. BONIS, P.A.; FRIEDMAN, S.L.; KAPLAN, MM. 2001. Is liver fibrosis reversible? New Engl. J. Med. 344:452-454.

9. BROWN, A.J.; DAVISON, E.; SLEEPER, M.M. 2010. Clinical efficacy of sildenafil in treatment of pulmonary arterial hipertension in dogs. J. Vet. Intern. Med. 24:850-854.

10. COLLE, I.; DE VRIESE, A.S.; VAN VLIERBERGHE, H.; LAMEIRE, N.H.; DEVOS, M. 2004. Systemic and splanchnic haemodynamic effects of sildenafil in an in vivo animal model of cirrhosis support for a risk in cirrhotic patients. Liver Int. (Reino Unido). 24:63-68.

11. CORBIN, J.; FRANCIS, S.; WEBB, D. 2002. Phosphodiesterase type 5 as a pharmacologic target in erectile dysfunction. Urology (Estados Unidos). 60(Supplement 2B):4-11.

12. CRUZ-AMAYA, J.; NARVÁEZ, N.; URREA, M.; FLOREZ, M.; MEZA, H. 2011. Efecto de la aplicación de dexametasona en la evolución de la enfermedad hepática crónica, en un modelo de experimentación Murino. Rev. Lasallista Investig. (Colombia). 8(2):33-39.

13. DUMITRESCU, D.; SECK, C.; MÖHLE, L.; ERDMANN, E.; ROSENKRANZL, S. 2012. Therapeutic potential of sildenafil in patients with heart failure and reactive pulmonary hypertension. Int. J. Cardiol. (Holanda). 154(2):205-206.

14. EDWARDS, C.; FENG, H-Q.; REYNOLDS, C.; MAO, L.; ROCKEY, D. 2008. Effect of the nitric oxide donor V-PYRRO/NO on portal pressure and sinusoidal dynamics in normal and cirrhotic mice. Am. J. Physiol. Gastrointest. Liver Physiol. 294:G1311-G1317.

15. FAILLI, P.; DEFRANCO, R.; CALIGIURI, A.; GENTILINI, A.; ROMANELLI, R.; MARRA, F.; BATIGNANI, G.; GUERRA, C.; LAFFI, G.; GENTILINI, P.; PINZANI, M. 2000. Nitrovasodilators inhibit platelet-derived growth factor induced proliferation and migration of activated human hepatic stellate cells. Gastroenterol. (Estados Unidos). 119:479-492.

16. FORBES, S.; PAROLA, M. 2011. Liver fibrogenic cells. Best Pract. Res. Clin. Gastroenterol. (Reino Unido). 25(2):207-217.

17. GHALI-GHOUL, R.; TAHSELDAR-ROUMIEH, R.; SABRA, R. 2007. Effect of chronic administration of sildenafil on sodium retention and on the hemodynamic complications Associated with Liver Cirrhosis in the Rat. Eur. J. Pharmacol. (Paises Bajos). 572:49-56. 
18. HALCOX, J.; NOUR, A.; ZALOS, G.; MINCEMOYER, R.; WACLAWIW, A.; RIVERA, E.; WILLIE, G.; ELLAHHAM, S.; QUYYUMI A. 2002. The Effect of Sildenafil on human vascular function, platelet activation, and myocardial ischemia. J. Am. Coll. Cardiol. 40:12321240.

19. IREDALE, J. 2003. Cirrhosis: New research provides a basis for rational and targeted treatments. Brit. Med. J. 327:143-147.

20. JI, H.; SHEN, H.; UHANOVA, J.; ZHANG, M.; MINUK, G.; GONG, Y. 2005. Effects of sildenafil citrate on hepatic function and regeneration in normal and alcohol-fed rats. Liver Int. 25:913-919.

21. KELLUM, H.B.; STEPIEN, R.L. 2007. Sildenafil citrate therapy in 22 dogs with pulmonary hypertension. J. Vet. Intern. Med. 21(6):1258-1264.

22. LI, J.; FU, P.; DELEON, M.; FRENCH, B.A.; FRENCH, S.W. 2005. The effect of viagra (sildenafil citrate) on liver injury caused by chronic ethanol intragastric feeding in Rats. Exp. Mol. Pathol. (Estados Unidos) 78:101-108.

23. LOUREIRO-SILVA, M.; IWAKIRI, Y.; ABRALDES, J.; HAQ, O.; GROSZMANN, R. 2006. Increased phosphodiesterase- 5 expression is involved in the decreased vasodilatador response to nitric oxide in cirrhotic rat livers. J. Hepatol. 44:886-893.

24. MAYORAL, P.; CRIADO, M.; HIDALGO, F.; FLORES, O.; ARÉVALO, A.; ELENO, N.; SÁNCHEZ- RODRÍGUEZ, A.; LÓPEZ-NOVOA, M.; ESTELLER, A. 1999. Effects of chronic nitric oxide activation or inhibition on early hepatic fibrosis in rats with bile duct ligation. Clin. Sci. (Reino Unido). 96(3):297-305.

25. OLIVER, J.; MELVILLE, V.; WEBB, D. 2006. Effect of regular phosphodiesterase type 5 inhibition in hypertension. Hypertension Ann. $\mathrm{H}$ (Estados Unidos). 48:622-627.

26. PROCTOR, E.; CHATAMRA, K. 1984. Standardized micronodular cirrhosis in the rat. Eur. Surg. Res. 16:182-186.

27. REYNA-VILLASMIL, E.; TORRE, D.; PEÑA, E.; MEJÍA, J.; REYNA-VILLASMIL, N.; GONZÁLEZ, P. 2008. Efecto del citrato de sildenafil sobre el flujo sanguíneo de las arterias uterina, umbilical y cerebral media fetal en pre eclámpticas. Rev. Obstetr. Ginecol. Ven. 68:187194.
28. ROCKEY, D.; SHAH, V. 2004. Nitric oxide biology and the liver: Report of an AASLD research workshop. Hepatology (Estados Unidos). 39:121-128.

29. RODRÍGUEZ-VILARRUPLA, A.; FERNÁNDEZ, M.;BOSCH, J.; GARCÍA-PAGÁN, C. 2007. Current concepts on the pathophysiology of portal hypertension. Ann. Hepatol. (Estados Unidos). 6(1):28-36.

30. SHENOY, P.; AGARWAL, V. 2010. Phosphodiesterase inhibitors in the management of autoimmune disease. Autoimmun. Rev. (Holanda). 9(7):511-515.

31. SPRING, R.M.; ULRICH, S.; HUBER, L.C.; SPEICH, R.; MAGGIORINI M.; TREDER, U.; FISCHLER, $M$. 2008. Sildenafil for pulmonary hypertension: dosedependent improvement in exercise performance. Pulm. Pharmacol. \& Ther. (Estados Unidos). 21:516521.

32. TANDON, P.; INAYAT, I.; TAL, M.; SPECTOR, M.; GROSZMANN, R.; GARCIA-TSAO, G. 2010. Sildenafil has no effect on portal pressure but lowers arterial pressure in patients with compensated cirrhosis. Clin. Gastroenterol. 8:546-549.

33. THEISE, N. 2007. Liver biopsy assessment in chronic viral hepatitis. Modern Pathol. (Estados Unidos). 20:3-14.

34. TOMASONI, L.; SITIA, S.; BORGHI, C.; CICERO, A.; CECONI, C.; CECARO, F.; MORGANTI, A.; DE GENNARO COLONNA, V.; GUAZZI, M.; MORRICONE, L.; MALAVAZOS, A.E.; MARINO, P.; CAVALLINO, C.; SHOENFELD, Y.; TURIE, M. 2010. Effects of treatment strategy on endothelial function. Autoimmun. Rev. 9(12):840-844.

35. YARDIMCI, S.; BOSTANCI, E.B.; OZER, I.; DALGIC, T.; SURMELIOGLU, A.; AYDOG, G.; AKOGLU, M. 2012. Sildenafil accelerates liver regeneration after partial hepatectomy in rats. Transplant Proc. (Estados Unidos). 44:1747-1750.

36. YILDIRIM, A.; ERSOY, Y.; ERCAN, F.; ATUKEREN, P.; GUMUSTAS, K.; USLU, U.; ALICAN, I. 2010. Phosphodiesterase- 5 inhibition by sildenafil citrate in a rat model of bleomycin-induced lung fibrosis. Pulm. Pharmacol. Ther. (Estados Unidos). 23:215221.

Recibido: Abril 5 de 2012

Aceptado: Enero 21 de 2013 